\title{
On extracting information about hadron-nuclear interaction from hadronic atom level shifts
}

\author{
J. Révai* \\ Research Institute for Particle and Nuclear Physics, \\ H-1525 Budapest, P.O.B. 49, Hungary \\ N.V. Shevchenko \\ Nuclear Physics Institute, $25068 \check{R} e \check{z}$, Czech Republic
}

(Dated: March 9, 2022)

\begin{abstract}
It is argued, that adjusting strong potentials directly to observed hadronic atom level shifts may lead to significantly different scattering lengths, than those, predicted by the Deser formula [1]. On the example of the 1s level shift of kaonic hydrogen it is demonstrated, that the usually adopted Deser values deduced from the two recent measurements in KEK [2] and by the DEAR Collaboration [3] $a_{D}(\mathrm{KEK})=0.78-0.49 i \mathrm{fm}$ and $a_{D}(\mathrm{DEAR})=0.47-0.3 i \mathrm{fm}$ should be replaced by $a_{s}(\mathrm{KEK}) \simeq 0.85-0.62 i \mathrm{fm}$ and $a_{s}(\mathrm{DEAR}) \simeq 0.49-0.35 i \mathrm{fm}$, correspondingly.
\end{abstract}

\footnotetext{
* Corresponding author: revai@rmki.kfki.hu
} 


\section{INTRODUCTION}

One of the most relevant sources of information on hadron-nucleus interaction is the measurement of hadronic atom level shifts. The usual picture of a hadronic atom is a particle moving in the combined Coulomb and nuclear potential of the nucleus.(see e.g. [4]). The measured level shifts are used to extract information about the nuclear part of the potential.

An important subfield is the study of hadronic hydrogen, which gives information on the primary hadron nucleon interaction. According to the usual philosophy of hadronic atoms, this interaction is imagined as a complex potential, the properties of which are to be deduced (at least partly) from the measured level shifts. On the other hand, the elementary hadron nucleon interaction can be approached also from the field-theoretical side, trying to derive it from effective Lagrangians and then to relate it to the level shifts [5]. While the field theoretical approach can be considered as more fundamental in this case, the basic merit of the potential picture leading to a phenomenological potential lies in its applicability in dynamical description of more complicated $N>2$ systems in the quantum mechanical framework. Proper field theoretical calculations of such systems are still beyond the real possibilities.

In both cases the level shifts and the nuclear interactions are related via the scattering length of the nuclear interaction. The use of scattering length seems to be natural in the field-theoretical case since it is the zero energy scattering amplitude and thus diagram technique can be applied for its calculation. On the other hand, in the potential approach this intermediate step seems to be superfluous, with present day computational facilities the potentials can be directly and easily related to the level shifts.

The main purpose of the paper is to show, that the approximate deduction of the potential parameters via the widely used Deser-formula [1], connecting the level shift and the scattering length can lead to substantial differences, compared to the direct approach (this has been known before). We argue, that the effort of exact solution of the Schrödinger-equation, yielding accurate potential parameters is not greater, than that of applying different corrections to the Deser-formula. Also, a new approximate relation between the level shifts and the potentials is derived, which practically gives the exact results.

As a demonstration of the above ideas we have made calculations for the the kaonic 
hydrogen case since recently the $\bar{K} N$ interaction has attracted some renewed interest due to the possible existence of bound $\bar{K}$-nuclear states.

\section{FORMULATION OF THE PROBLEM}

Let us consider the $1 s$ level of kaonic hydrogen. The (model) Hamiltonian reads

$$
H=-\frac{\hbar^{2}}{2 \mu} \Delta_{\mathbf{r}}+V_{s}+V_{c}
$$

where the strong interaction is represented by a local central potential

$$
V_{s}=V_{0} v_{s}(r / b)
$$

with two parameters: its strength $V_{0}$ and range $b$. The attractive Coulomb potential is

$$
V_{c}=-\frac{e^{2}}{r}
$$

and $\mu$ is the $K^{-} p$ reduced mass:

$$
\mu=323.478 \mathrm{MeV} / \mathrm{c}^{2}
$$

The Schrödinger equation $(H-E) \Psi=0$ can be transformed into the radial equation

$$
\left(\frac{d^{2}}{d r^{2}}-q^{2}-\lambda v_{s}(r / b)+\frac{2 / r_{0}}{r}\right) \psi(r)=0,
$$

where

$$
E=-\frac{\hbar^{2}}{2 \mu} q^{2}, \quad \lambda=\frac{2 \mu}{\hbar^{2}} V_{0}
$$

and $r_{0}$ is the Bohr radius

$$
r_{0}=\frac{\hbar^{2}}{\mu e^{2}}=83.594 \mathrm{fm}
$$

The "atomic" energy unit in this case is

$$
\varepsilon_{0}=\frac{\mu e^{4}}{\hbar^{2}}=17.226 \mathrm{keV}
$$

In connection with Eq. (5) we are interested in the change of the eigenvalue $q^{2}$ compared to the $1 s$ eigenvalue $q_{0}^{2}$ of the pure Coulomb equation

$$
\left(\frac{d^{2}}{d r^{2}}-q_{0}^{2}+\frac{2 / r_{0}}{r}\right) \varphi(r)=0 .
$$

The solution of Eq. (6) is, of course, known:

$$
q_{0}=\frac{1}{r_{0}}, \quad \varphi \sim \frac{r}{r_{0}} \exp \left(-\frac{r}{r_{0}}\right) .
$$

Let us recall some important features of Eq. (5): 
- the strong interaction acts on the fm scale and is vanishingly small beyond a certain distance $R: b \sim \mathrm{fm}, v_{s}(r / b) \sim 0$ for $r>R$;

- due to absorbtion to other channels, $\lambda$ is complex, so $q$ is complex, too, but $\operatorname{Re}(q)>0$, so $\psi(r) \rightarrow 0$ for $r \rightarrow 0$;

- both functions $\psi(r)$ and $\varphi(r)$ are on the $r_{0}$ scale $(\sim 100 \mathrm{fm})$, while the presence of $V_{s}$ modifies $\psi(r)$ compared to $\varphi(r)$ only for $r<R$; thus $q^{2}-q_{0}^{2}$ is small, but not due to the smallness of $V_{s}$ itself, so perturbative treatment is not justified;

- outside the range of the strong potential $(r>R) \psi(r)$ goes over into the asymptotically vanishing solution of the Coulomb equation

$$
\psi(r) \sim F_{\text {out }}^{c}(q, r)=r \exp (-q r) U\left(1-\frac{1}{q r_{0}}, 2 ; 2 q r\right)
$$

with $U$ being the confluent hypergeometric function of the second kind [6].

- within the range of $V_{s}(r<R)$ the relation $q^{2} \ll \lambda v_{s}(r / b)$ usually holds since the Coulombic eigenvalue is in the $\mathrm{keV}$ range, while the nuclear potential is of $\mathrm{MeV}$ order; this feature allows to approximate $\psi(r)$ in this range by the zero-energy solution $\psi^{0}(r)$.

Multiplying Eq. (5) by $\varphi(r)$ and Eq. (6) by $\psi(r)$, subtracting and integrating, we obtain in the usual way:

$$
q^{2}-q_{0}^{2}=-\frac{\int_{0}^{R} \varphi(r) \lambda v_{s}(r / b) \psi(r) d r}{\int_{0}^{\infty} \varphi(r) \psi(r) d r},
$$

where we used the fact, that both $\psi(r)$ and $\varphi(r)$ vanish for $r \rightarrow \infty$ and $r \rightarrow 0$.

The expression (77) is exact and independent of the normalization of $\psi(r)$ and $\varphi(r)$. The basic question is how to relate

$$
\Delta E=-\frac{\hbar^{2}}{2 \mu}\left(q^{2}-q_{0}^{2}\right)
$$

to the properties of $V_{s}$.

The traditional answer to this question was given by Deser [1], back in 1954. According to him

$$
\begin{array}{r}
q^{2}-q_{0}^{2} \approx-4 \frac{a_{s}}{r_{0}^{3}} \quad \text { or } \\
a_{D}(q)=-\frac{r_{0}^{3}}{4}\left(q^{2}-q_{0}^{2}\right) \approx a_{s},
\end{array}
$$


where $a_{s}$ is the scattering length of the strong potential $V_{s}$ defined as ${ }^{1}$

$$
a_{s}=\frac{\int_{0}^{R} r \lambda v_{s}(r / b) \psi_{s}^{0}(r) d r}{1+\int_{0}^{R} \lambda v_{s}(r / b) \psi_{s}^{0}(r) d r}=R-\frac{\psi_{s}^{0}(R)}{\psi_{s}^{0^{\prime}}(R)},
$$

with $\psi_{s}^{0}(r)$ being the regular $\left[\psi_{s}^{0}(0)=0, \psi_{s}^{0^{\prime}}(0)=1\right]$ solution of the zero-energy equation with $V_{s}$ alone:

$$
\left(\frac{d^{2}}{d r^{2}}-\lambda v_{s}(r / b)\right) \psi_{s}^{0}(r)=0 .
$$

In other words, the pure strong scattering length is approximated by the Deser scattering length $a_{D}(q)$, derived from the measured $\Delta E$.

It is not straightforward to relate (8) and (9) to the exact expression (77) or to point out clearly the approximations leading from (77) to (8), together with criteria for their applicability (apart from the obvious $R \ll r_{0}$ ).

Since 1954 considerable effort has been devoted to re-derivation of Deser's result, to considering its possible improvements or corrections to it, to studying its special cases, e.g. a strong potential with an almost or weakly bound state, when the scattering length becomes large [7]-[11]. The most relevant improvement is the taking into account the Coulomb distortion of the zero-energy wave function $\psi_{s}^{0}$, leading to approximation

$$
a_{D}(q) \approx a_{s c}
$$

instead of (8), where $a_{s c}$ is the Coulomb modified scattering length:

$$
a_{s c}=\frac{\int_{0}^{R} \Phi(r) \lambda v_{s}(r / b) \psi_{s c}^{0}(r) d r}{1+\int_{0}^{R} \Theta(r) \lambda v_{s}(r / b) \psi_{s c}^{0}(r) d r}=\frac{\left.W\left(\Phi, \psi_{s c}^{0}\right)\right|_{r=R}}{\left.W\left(\Theta, \psi_{s c}^{0}\right)\right|_{r=R}} .
$$

Here $\Phi(r)$ and $\Theta(r)$ are the suitable $q \rightarrow 0$ limits of the Coulomb scattering functions $F$ and $G$ (see [12]), satisfying

$$
\left(\frac{d^{2}}{d r^{2}}+\frac{2 / r_{0}}{r}\right)\left\{\begin{array}{l}
\Phi(r) \\
\Theta(r)
\end{array}\right\}=0
$$

and $\psi_{s c}^{0}(r)$ is the regular zero-energy solution of

$$
\left(\frac{d^{2}}{d r^{2}}-\lambda v_{s}(r / b)+\frac{2 / r_{0}}{r}\right) \psi_{s c}^{0}(r)=0
$$

\footnotetext{
1 This is the usual textbook definition of $a_{s}$ corresponding to negative scattering length for weak attractive potential $(\lambda<0)$. By some reason in meson-nuclear physics the opposite sign is used.
} 
while $W$ denotes the wronskian of the two functions.

Now, the usual way, how (8) and (11) are used to relate a strong potential $V_{s}$ to the measured energy shift is to approximate $a_{s}\left(\right.$ or $\left.a_{s c}\right)$ by $a_{D}(q)$ and then to design a potential $V_{s}$ having this $a_{s}\left(\right.$ or $\left.a_{s c}\right)$ :

$$
\Delta E \Rightarrow q^{2}-q_{0}^{2} \Rightarrow a_{D}(q) \Rightarrow \begin{aligned}
& a_{s} \approx a_{D}(q) \\
& a_{s c} \approx a_{D}(q)
\end{aligned} \Rightarrow V_{s}
$$

But due to the approximations (8) or (11) the $\Delta E^{\prime}$ values, calculated with the potentials obtained in this way do not reproduce the measured $\Delta E$-s : $\Delta E^{\prime} \neq \Delta E$, so the usual experimental claim, that measuring $\Delta E$ is equivalent to measuring $a_{s}$ is not valid.

However, at the present calculational level $(2007 \gg 1954)$ to find a potential, giving a prescribed $a_{s}$ (or $a_{s c}$ ) is not easier, than to find a potential giving exactly the measured $\Delta E$. The eigenvalue equation (5) is solved by numerical integration in the internal region $(r \leq R)$ and by matching the logarithmic derivatives of $\psi_{\text {in }}(r)$ and the external function $F_{\text {out }}^{c}(q, r)$ at $r=R$ :

$$
\frac{\psi_{\text {in }}{ }^{\prime}(R)}{\psi_{\text {in }}(R)}-\frac{F_{\text {out }}^{c}{ }^{\prime}(q, R)}{F_{\text {out }}^{c}(q, R)}=A(\lambda, q)=0 .
$$

For a given range parameter $b$ the root in $q$ of Eq. (15) for fixed $\lambda$ gives the eigenvalue, while the root in $\lambda$ for fixed $q$ yields the potential strength corresponding to a prescribed eigenvalue. The numerical solution of Eq. (15) is straightforward in both cases.

For demonstration of the above idea strong potentials were derived from the three conditions

$$
\begin{gathered}
\Delta E(\text { calc })=\Delta E(\exp ) \\
a_{s}=a_{D}\left(q_{\exp }\right) \\
a_{s c}=a_{D}\left(q_{\exp }\right)
\end{gathered}
$$

their level shifts and strong scattering lengths were compared for four type of commonly used potential shapes: exponential, gaussian, square well, and Yamagouchi (non-local, separable) and different ranges $b$. The experimental $\Delta E(\exp )$ was taken from the KEK experiment [2]:

$$
\begin{gathered}
\Delta E(\mathrm{KEK})=-323+203.5 i \mathrm{eV} \text { with } \\
a_{D}(\mathrm{KEK})=0.78-0.49 i \mathrm{fm} .
\end{gathered}
$$




\section{RESULTS AND CONCLUSIONS}

The main message of this work is to emphasize, that no approximations are needed to connect the measured $\Delta E(\exp )$ with the properties of the strong interaction (model) potential $V_{s}$. However, the desire to find a relation between these quantities without solving the eigenvalue equation, which is superior to the previously used ones, motivated the derivation of another approximate formula.

The normalization integral in the denominator of Eq. (7) can be approximated as

$$
\begin{aligned}
\int_{0}^{\infty} \varphi(r) \psi(r) d r \approx & \int_{R}^{\infty} \varphi(r) \psi(r) d r= \\
& \int_{R}^{\infty} \varphi(r) F_{\text {out }}^{c}(q, r) d r
\end{aligned}
$$

due to the smallness of the nuclear region $0<r<R$ compared to the whole range of functions $\varphi(r)$ and $\psi(r)$. Using the equations satisfied by $\varphi(r)$ and $F_{\text {out }}^{c}(r)$ and their vanishing for $r \rightarrow \infty$ Eq. (17) can be rewritten as

$$
\int_{R}^{\infty} \varphi(r) F_{\text {out }}^{c}(q, r) d r=\frac{\left.W\left(F_{\text {out }}^{c}, \varphi\right)\right|_{r=R}}{q^{2}-q_{0}^{2}} .
$$

Substituting (18) into (17) and (7) and, as before, approximating $\psi(r)$ within the nuclear range by $\psi_{s c}^{0}(r)$ we get finally

$$
\left.W\left(F_{\text {out }}^{c}, \varphi\right)\right|_{r=R}=-\int_{0}^{R} \varphi(r) \lambda v_{s}(r / b) \psi_{s c}^{0}(r) d r,
$$

which is of the form

$$
w(q)=I(\lambda)
$$

and again can be solved either for $q$ or for $\lambda$. The results for the strong potentials obtained from condition $(d)$ are also shown in Table 1 , summarizing our results.

From these results we can make the following conclusions:

(i) The quality of approximations

It can be seen, that the most commonly used approximation (b) gives rather poor results, in the considered case the error in $\Delta E^{\prime}$ is of the order of $10-15 \%$. The opposite is also true: the $a_{s}$-s corresponding to potentials exactly reproducing the measured $\Delta E$ differ from the Deser value $a_{D}$ approximately by the same amount. The improved approximation $(c)$ yields better strong potentials, the error both in $\Delta E^{\prime}$ and 
$a_{s}$ amounts to a few $\%$. The best results seem to be given by approximation $(d)$, which practically reproduces the exact $\Delta E$-s and $a_{s}$-s of the exact calculation $(a)$.

(ii) Model independence

One of the most attractive features of the Deser formula is its model independence: the relation between the energy shift and the scattering length is independent of the form of the potential. This feature is confirmed by the calculations with different potential shapes and quite different ranges. The relation between $\Delta E$ and $a_{s}$ is almost potential independent, however, only within one approximation.

(iii) Finally, to the question ,,which feature of a given potential instead of $a_{s}$ or $a_{s c}$ determines $\Delta E$ ? ", our answer is:

$$
I(\lambda)=-\int_{0}^{R} \varphi(r) \lambda v_{s}(r / b) \psi_{s c}^{0}(r) d r
$$

through the relation $(d)$.

(iv) The widely used ,measured "values of $a_{s}(\mathrm{KEK})$ for the kaonic hydrogen should be changed from $a_{D}\left(q_{\exp }\right)$ (16) to

$$
a_{s}(\mathrm{KEK}) \simeq 0.85-0.62 i \mathrm{fm}
$$

since this is the value, which corresponds to potentials exactly reproducing the measured level shift.

The same calculation was also performed for the case of the DEAR $1 s$ level shift [3], and while the overall results are qualitatively the same as before, the correct scattering length corresponding to the DEAR shift is

$$
a_{s}(\mathrm{DEAR}) \simeq 0.49-0.35 i \mathrm{fm}
$$

instead of the widely adopted $a_{D}(\mathrm{DEAR})=0.47-0.30 i \mathrm{fm}$.

\section{Acknowledgments}

The work was supported by the Czech GA AVCR grant A100480617.

[1] Deser, S., Baumann, K., Thirring, W., Phys. Rev. 96, (1954) 774. 
[2] Ito, T. M. et al., Phys. Rev. C 58, (1998) 2366.

[3] Beer, G. et al., Phys. Rev. Lett. 94, (2005) 212302.

[4] Deloff, A.: Fundamentals in Hadronic Atom Theory, World Scientific, Singapore, 2003.

[5] Meissner, U.-G., Raha, U., Rusetsky, A., Eur. Phys. J. C 35, (2004) 349.

[6] Abramowitz, M., Stegun, I. A.: Handbook of Mathematical Functions, 10th ed.; Dover Publications, New York 1972, p. 504.

[7] Trueman, T. L., Nucl. Phys. 26, (1976) 57.

[8] Deloff, A., Phys. Rev. C 13, (1976) 730.

[9] Thaler, J., J. Phys. G 9, (1983) 1009.

[10] Mitroy, J. and Ivallov, A., J. Phys. G 27,(2001) 1421.

[11] Popov, V. S. et al., Sov. Phys. JETP 53, (1981) 650;

Zh. Exp. Teor. Fiz. 80, (1981) 1271.

[12] Lambert, E., Helv. Phys. Acta 42, (1969) 667. 
TABLE I: Calculated kaonic hydrogen $1 s$ level shifts $\Delta \mathrm{E}$ and scattering lengths $a_{s}$ of different strong interaction potentials, derived from the measured KEK level shift $\Delta E(\exp )=$ $-323+203.5 i \mathrm{eV}$ using the conditions $(a)-(d)$ (see the text).

\begin{tabular}{|c|c|c|c|c|c|c|c|c|}
\hline & \multicolumn{2}{|c|}{ Gauss } & \multicolumn{2}{|c|}{ Exponential } & \multicolumn{2}{|c|}{ Square well } & \multicolumn{2}{|c|}{ Yamaguchi } \\
\hline$b, \mathrm{fm}$ & $a_{s}, \mathrm{fm}$ & $\Delta \mathrm{E}, \mathrm{eV}$ & $a_{s}, \mathrm{fm}$ & $\Delta \mathrm{E}, \mathrm{eV}$ & $a_{s}, \mathrm{fm}$ & $\Delta \mathrm{E}, \mathrm{eV}$ & $a_{s}, \mathrm{fm}$ & $\Delta \mathrm{E}, \mathrm{eV}$ \\
\hline \multicolumn{9}{|c|}{$V_{s}$ from $\Delta \mathrm{E}($ calc $)=\Delta \mathrm{E}(\exp )$} \\
\hline 1.0 & $0.85-0.61 i$ & & $0.88-0.61 i$ & & $0.84-0.61 i$ & & $0.86-0.61 i$ & \\
\hline 0.5 & $0.84-0.62 i$ & $-323+204 i$ & $0.85-0.61 i$ & $-323+204 i$ & $0.84-0.62 i$ & $-323+204 i$ & $0.84-0.61 i$ & $-323+204 i$ \\
\hline 0.25 & $0.84-0.63 i$ & & $0.84-0.62 i$ & & $0.84-0.64 i$ & & $0.84-0.62 i$ & \\
\hline \multicolumn{9}{|c|}{$V_{s}$ from $a_{s}=a_{D}$} \\
\hline 1.0 & & $-298+168 i$ & & $-289+167 i$ & & $-299+167 i$ & & $-294+168 i$ \\
\hline 0.5 & $0.78-0.49 i$ & $-299+165 i$ & $0.78-0.49 i$ & $-296+168 i$ & $0.78-0.49 i$ & $-299+164 i$ & $0.78-0.49 i$ & $-298+167 i$ \\
\hline 0.25 & & $-299+162 i$ & & $-298+166 i$ & & $-298+161 i$ & & $-300+165 i$ \\
\hline
\end{tabular}

$1.0 \quad 0.83-0.57 i-317+191 i 0.86-0.57 i-317+191 i 0.83-0.57 i-317+191 i 0.84-0.58 i-317+191 i$

$0.50 .83-0.58 i-317+191 i 0.84-0.57 i-317+191 i 0.83-0.58 i-317+191 i 0.83-0.57 i-317+191 i$ $0.250 .83-0.59 i-317+191 i 0.83-0.58 i-317+191 i 0.83-0.60 i-317+191 i 0.83-0.56 i-317+191 i$

$V_{s}$ from $w(q)=I(\lambda)$

$1.0 \quad 0.85-0.61 i-323+204 i 0.86-0.61 i-319+202 i 0.84-0.61 i-323+204 i 0.86-0.61 i-323+204 i$

$0.50 .84-0.62 i-323+204 i 0.86-0.61 i-319+202 i 0.84-0.62 i-322+204 i 0.84-0.61 i-322+203 i$

$0.250 .84-0.63 i-323+204 i 0.85-0.61 i-322+203 i 0.84-0.63 i-323+204 i 0.84-0.62 i-323+203 i$ 